\title{
Approximating the Volume of Convex Bodies
}

\author{
U. Betke and M. Henk \\ Mathematisches Institut, Universität Siegen, Hölderlinstrasse 3, \\ W-5900 Siegen, Federal Republic of Germany \\ henk@hrz.uni-siegen.dbp.de
}

\begin{abstract}
It is a well-known fact that for every polynomial-time algorithm which gives an upper bound $\vec{V}(K)$ and a lower bound $V(K)$ for the volume of a convex set $K \subset E^{d}$ given by an oracle, the ratio $\bar{V}(K) / \underline{V}(K)$ is at least $(c d / \log d)^{d}$. Here we describe an algorithm which gives, for $\varepsilon>0$, in polynomial time, an upper and lower bound with the property $\bar{V}(K) / \underline{V}(K) \leq d !(1+\varepsilon)^{d}$.
\end{abstract}

\section{Introduction}

Since it is hard to compute the volume of convex bodies in high dimensions, polynomial deterministic algorithms which give an upper bound $\bar{V}(K)$ and a lower bound $\underline{V}(K)$ for the volume $V(K)$ of a $d$-dimensional convex body $K$ may be requested. Indeed, such algorithms were given by Lovász (see, e.g., p. 122 of [GLS]) with a ratio $\bar{V}(K) / \underline{V}(K) \leq d^{3 d / 2}$ and by Applegate and Kannan [AK] quoted in $[D F]$-with $\bar{V}(K) / \underline{V}(K) \leq 2^{d} \cdot d !\left(1+1 / d^{2}\right)^{d}$. Here we give an algorithm which computes, for any $\varepsilon>0$, in polynomial time, $\bar{V}(K), \underline{V}(K)$ such that $\bar{V}(K) / \underline{V}(K) \leq$ $d !(1+\varepsilon)^{d}$.

The bounds given above appear to be very weak. However, Bárány and Füredi [BF] showed-see also [E] - that, for any polynomial deterministic algorithm, the ratio $\bar{V}(K) / \underline{V}(K)$ is at least $(c d / \log d)^{d}=d^{d(1-o(1))}$ for some constant $c$ independent of $d$ and we have, by Stirling's formula, $d !=\sqrt{2 \pi d}(d / e)^{d}(1-o(1))$.

Algorithms of this kind are not only of interest in their own sake but also because the newly devised randomized algorithms for computing the volume need good inscribed and circumscribed parallelepipedra (see, e.g., [DF] and [DFK]). Such parallelepipedra can be constructed from the bodies obtained by our algorithm.

Thus it seems to be worth while not only to show polynomiality but also to compare the running times somewhat closer. Here it turns out that the running time-in a sense made more precise below - of our algorithm is $1 / d^{2}$ of the algorithm of Applegate and Kannan. 
To make our ideas more precise we need some notation and we must state how the convex bodies are given: Let $E^{d}$ denote the $d$-dimensional euclidean space and the set of all convex bodies-compact convex sets-in $E^{d}$ is denoted by $\mathscr{K}^{d}$. $e^{i}$ denotes the $i$ th canonical unit vector and, for a vector $x \in E^{d}$, the $i$ th coordinate is denoted by $x_{i}$. Further, $\|\cdot\|$ denotes the euclidean norm, $\|\cdot\|_{\infty}$ denotes the maximum norm, and, for $y^{1}, \ldots, y^{d} \in E^{d}$, the determinant of the matrix with column vectors $y^{1}, \ldots, y^{d}$ is denoted by $\operatorname{det}\left(y^{1}, \ldots, y^{d}\right)$. Finally, $L\left(y^{1}, \ldots, y^{i}\right)$ denotes, for $y^{1}, \ldots, y^{i} \in E^{d}$, the linear space spanned by $y^{1}, \ldots, y^{i}$.

For the description of a convex body $K$ we adopt the oracle model as studied in detail in [GLS]. This means that a convex body $K \in \mathscr{K}^{d}$ is given by a so-called weak membership oracle WMEMO. This is a black box with the following properties:

Given a point $y \in \mathbb{Q}^{d}$ and a positive rational number $\varepsilon \in \mathbb{Q}$, the oracle answers that $y \in S(K, \varepsilon)$ or that $y \notin S(K,-\varepsilon)$,

where $S(K, \varepsilon)=\left\{x \in E^{d} \mid\|x-y\| \leq \varepsilon\right.$ for some $\left.y \in K\right\}$ and

$$
S(K,-\varepsilon)=\left\{x \in E^{d} \mid S(x, \varepsilon) \subset K\right\} .
$$

Moreover, we must make the assumption that we have the following information about the convex body $K$, given by a WMEMO: two rational numbers $R$, $r>0$ and a point $a \in \mathbb{Q}^{d}$ with $S(a, r) \subset K \subset S(0, R)$. For simplification we may assume

$$
S(0, r) \subset K \subset S(0, R)
$$

By using a special version of the ellipsoid method Yudin and Nemirovskii [YN], [GLS, p. 107] showed that there exist oracle-polynomial-time algorithms that solve the following problems for a convex body given by a WMEMO and (1.1):

(1) The weak violation problem (WVIOL):

Given a vector $c \in \mathbb{Q}^{d}$ and rational numbers $\gamma, \varepsilon, \varepsilon>0$, either

assert that $c^{T} x \leq \gamma+\varepsilon$ for all $x \in S(K,-\varepsilon)$, or

find a vector $y \in S(K, \varepsilon)$ with $c^{T} y \geq \gamma-\varepsilon$.

(2) The weak optimization problem (WOPT):

Given a vector $c \in \mathbb{Q}^{d}$ and a rational number $\varepsilon>0$, either

find $y \in \mathbb{Q}^{d}$ such that $y \in S(K, \varepsilon)$ and $c^{T} x \leq c^{T} y+\varepsilon$ for all $x \in S(K,-\varepsilon)$, or

assert that $S(K,-\varepsilon)$ is empty.

The result of Applegate and Kannan [AK] can now be stated as follows: Given a convex body $K \in \mathscr{K}^{d}$ by a WMEMO and (1.1), a parallelepiped $P$ and a simplex $S$ can be found, such that $S \subset K \subset P$ and $V(P) / V(S) \leq d ! 2^{d}\left(1+1 / d^{2}\right)^{d}$. Beside some elementary matrix operations the running time of this algorithm is dominated by at most $2 d^{3} \ln (2 d R / r)$ calls of the 
Briefly our algorithm can be described in the following way: we construct a parallelepiped which contains the given convex body $K$ and a polytope which is contained in $K$ such that the volume of the parallelepiped is not greater than $d$ ! times the volume of the inscribed polytope. To do this we need $2 d$ calls of the WOPT. Since we have no exact arithmetic our main result is

Theorem 1. There exists an oracle-polynomial-time algorithm that, for a convex body $K \in \mathscr{K}^{d}$ given by a WMEMO and (1.1) and for every $\varepsilon>0$, computes, by $2 d$ calls of the WOPT, an upper bound $\vec{V}(K)$ and a lower bound $\underline{V}(K)$ of the volume of $K$ such that

$$
\frac{\bar{V}(K)}{\underline{V}(K)} \leq d ! \cdot(1+\varepsilon)^{d} .
$$

Let us remark, that to compute, for a convex body $K \in \mathscr{K}^{d}$ given by a WMEMO and (1.1), nontrivial upper and lower bounds of the volume by using the WOPT we need at least $d+1$ calls of the WOPT: after $d$ calls of the WOPT we have the information that $K$ is contained in some unbounded polyhedron and contains $d$ points, which lie in a suitable affine hyperplane. Together with (1.1) we only get upper and lower bounds which depend on the input data $R, r$. From this point of view the running time of our algorithm is best possible up to a factor 2 .

Further, the running time of our algorithm - measured in the number of calls of the WVIOL -is approximately $1 / d^{2}$ of that of Applegate and Kannan as pointed out in the third part of this paper where the proof of Theorem 1 is given. In the second part we describe our algorithm in a more geometric form. From this presentation we deduce our basic theoretical result (Theorem 2). As a theoretical application of our algorithm we get an inequality connecting the volume of a convex body and certain successive diameters and widths. This result is indicated at the end of the second part and is a special case of a series of inequalities concerning successive diameters and widths, which are described in more detail in [BH].

\section{The Algorithm}

Geometric version. Let $K \in \mathscr{K}^{d}$.

(1) Let $c^{1} \in E^{d} \backslash\{0\}$ and $i=1$;

(2) Find $\bar{z}^{i}, \underline{z}^{i} \in K$ such that, for all $x \in K$,

$$
\left(c^{i}\right)^{T} \underline{z}^{i} \leq\left(c^{i}\right)^{T} x \leq\left(c^{i}\right)^{T} \bar{z}^{i}
$$

holds;

(3) let $y^{i}=\bar{z}^{i}-\underline{z}^{i}$;

(4) if $(i=d)$, then STOP;

(5) find $c^{i+1} \in E^{d} \backslash\{0\}$ such that $c^{i+1}$ is orthogonal to $L\left(y^{1}, \ldots, y^{i}\right)$;

(6) let $i=i+1$;

(7) GOTO (2). 
Theorem 2. Let $c^{i}, \bar{z}^{i}, \underline{z}^{i}, y^{i}, 1 \leq i \leq d$, satisfy: $c^{i} \neq 0, c^{i+1}$ orthogonal to $L\left(y^{1}, \ldots, y^{i}\right)$, $\bar{z}^{i}, \underline{z}^{i} \in K$ such that $\left(c^{i}\right)^{T} \underline{z}^{i} \leq\left(c^{i}\right)^{T} x \leq\left(c^{i}\right)^{T} \bar{z}^{i}$ for all $x \in K, y^{i}=\bar{z}^{i}-\underline{z}^{i}$. Then

$$
\frac{\left|\operatorname{det}\left(y^{1}, \ldots, y^{d}\right)\right|}{d !} \leq V(K) \leq\left|\operatorname{det}\left(y^{1}, \ldots, y^{d}\right)\right|
$$

Proof. Let $P$ be the parallelepiped given by

$$
P=\left\{x \in E^{d} \mid\left(c^{i}\right)^{T} \underline{z}^{i} \leq\left(c^{i}\right)^{T} x \leq\left(c^{i}\right)^{T} \bar{z}^{i}, 1 \leq i \leq d\right\}
$$

and let $C$ be the polytope with vertices $\underline{z}^{1}, \bar{z}^{1}, \ldots, \underline{z}^{d}, \bar{z}^{d}$. We obviously have $C \subset K \subset P$ and in the following we prove

$$
V(P)=\left|\operatorname{det}\left(y^{1}, \ldots, y^{d}\right)\right| \quad \text { and } \quad V(C) \geq\left|\operatorname{det}\left(y^{1}, \ldots, y^{d}\right)\right| / d !
$$

This is done by induction with respect to the dimension. For $d=1,(2.1)$ is trivial. Hence we may assume $d \geq 2$. Let $H=\left\{x \in E^{d} \mid\left(y^{1}\right)^{T} x=0\right\}$ and let $\bar{z}_{p}^{i}$, $\underline{z}_{p}^{i}$ be the images of the points $\bar{z}^{i}, \underline{z}^{i}$ under the orthogonal projection onto $H$, $1 \leq i \leq d$. Application of the Steiner symmetrization [BoF, p. 69] to $P$ and $C$ with respect to the hyperplane $H$ gives convex bodies $P_{S}, C_{S}$ with $V\left(C_{S}\right)=V(C)$ and $V\left(P_{S}\right)=V(P)$.

By definition of this symmetrization $C_{S}$ contains the polytope with vertices $\bar{z}_{p}^{1}+\frac{1}{2} y^{1}, \bar{z}_{p}^{1}-\frac{1}{2} y^{1}, \bar{z}_{p}^{2}, z_{p}^{2}, \ldots, \bar{z}_{p}^{d}, \underline{z}_{p}^{d}$ and hence we have

$$
V(C) \geq \frac{\left\|y^{1}\right\|}{d} \cdot V(\bar{C} ; H)
$$

where $\bar{C}$ is the polytope with vertices $\bar{z}_{p}^{2}, z_{p}^{2}, \ldots, \bar{z}_{p}^{d}, z_{p}^{d}$ and $V(\bar{C} ; H)$ denotes the volume of $\bar{C}$ with respect to the euclidean space $H$. On account of the choice of the directions $c^{i}$ we have

$$
P_{S}=\left\{x \in E^{d} \mid-\frac{\left\|y^{1}\right\|^{2}}{2} \leq\left(y^{1}\right)^{T} x \leq \frac{\left\|y^{1}\right\|^{2}}{2},\left(c^{i}\right)^{T} \underline{z}_{p}^{i} \leq\left(c^{i}\right)^{T} x \leq\left(c^{i}\right)^{T} \bar{z}_{p}^{i}, 2 \leq i \leq d\right\}
$$

and thereby

$$
V(P)=\left\|y^{1}\right\| \cdot V(\bar{P} ; H)
$$

with $\bar{P}=\left\{x \in H \mid\left(c^{i}\right)^{T} \underline{z}_{p}^{i} \leq\left(c^{i}\right)^{T} x \leq\left(c^{i}\right)^{T} \bar{z}_{p}^{i}, 2 \leq i \leq d\right\}$. Now the situation for $\bar{P}, \bar{C}$ in the space $H$ is the same as for $P, C$ and hence the assertion follows from (2.2) and (2.3) by using the induction hypothesis. 
Remark. A first way to choose the directions $c^{i}$ from a theoretical point of view is as follows: choose the $c^{i}$ in (1) and (5) such that the breadth in direction $c^{i}$ becomes minimal (maximal). If we do this it is easy to see that we can find $\bar{z}^{i}, z^{i}$ such that $L\left(c^{1}, \ldots, c^{i}\right)=L\left(y^{1}, \ldots, y^{i}\right)$. Further, $\left\|\bar{z}^{1}-\underline{z}^{1}\right\|$ gives the width (diameter) of the convex body and, for $i=2, \ldots, d$, the length of the projections of $\bar{z}^{i}-z^{i}$ onto the orthogonal complement of $L\left(y^{1}, \ldots, y^{i}\right)$ gives the width (diameter) of the projection of $K$ onto this space. Thus we obtain upper and lower bounds for the volume with respect to the product of "iterated" widths (diameters). This essentially proves the main theorem in $[\mathrm{BH}]$ for the case of projections.

\section{Proof of Theorem 1}

First we state the algorithm in its computational form.

Input: A rational number $\varepsilon>0$ and a convex body $K \in \mathscr{K}^{d}$ given by a WMEMO and (1.1)

Output: An upper bound $\bar{V}(K)$ and a lower bound $\underline{V}(K)$ of the volume of $K$ with the property $\bar{V}(K) / \underline{V}(K) \leq d !(1+\varepsilon)^{d}$.

[1] Let $\delta:=\min \{r / 3, \varepsilon r /(6+\varepsilon)\}, \alpha:=r /(r+\delta)$, and

$$
\beta:=\frac{r}{r-\delta}\left(1+\frac{\delta}{r-2 \delta}\right)
$$

[2] let $c^{1}:=e^{1}$ and $i:=1$;

[3] find $\bar{z}^{i}, \underline{z}^{i} \in S(K, \delta)$ such that, for all $x \in S(K,-\delta)$,

$$
\left(c^{i}\right)^{T} \underline{z}^{i}-\delta \leq\left(c^{i}\right)^{T} x \leq\left(c^{i}\right)^{T} \bar{z}^{i}+\delta
$$

holds;

[4] Let $y^{i}:=\bar{z}^{i}-\underline{z}^{i}$;

[5] if $(i=d)$, then

$$
\underline{V}(K):=\frac{\alpha^{d}\left|\operatorname{det}\left(y^{1}, \ldots, y^{d}\right)\right|}{d !} \text { and } \bar{V}(K):=\beta^{d}\left|\operatorname{det}\left(y^{1}, \ldots, y^{d}\right)\right|
$$

STOP.

[6] Find $c^{i+1} \in \mathbb{Q}^{d}$ such that $c^{i+1}$ is orthogonal to $L\left(y^{1}, \ldots, y^{i}\right)$ and $\left\|c^{i+1}\right\| \geq 1$;

[7] let $i:=i+1$;

[8] GOTO [3];

Proof of Theorem 1. First we study the correctness of the algorithm above. Since $S(0, r) \subset K$ we have, by simple geometric arguments $[r /(r+\delta)] x \in K$ for all $x \in S(K, \delta)$ and $[(r-\delta) / r] x \in S(K,-\delta)$ for all $x \in K$. Hence the polytope with 
vertices $\alpha \bar{z}^{1}, \alpha \underline{z}^{1}, \ldots, \alpha \bar{z}^{d}, \alpha z^{d}$ is contained in $K$. From (2.1),

$$
V(K) \geq \frac{\alpha^{d}}{d !} \cdot\left|\operatorname{det}\left(y^{1}, \ldots, y^{d}\right)\right|
$$

follows. On the other hand, we have, for all $x \in K$, the relations

$$
\frac{r}{r-\delta}\left(\left(c^{i}\right)^{T} \underline{z}^{i}-\delta\right) \leq\left(c^{i}\right)^{T} x \leq \frac{r}{r-\delta}\left(\left(c^{i}\right)^{T} \bar{z}^{i}+\delta\right), \quad 1 \leq i \leq d
$$

Since $\left\|c^{i}\right\| \geq 1$ and $S(0, r-\delta) \subset S(K,-\delta)$ we have $\left(c^{i}\right)^{T} \bar{z}+\delta \geq r-\delta$ and $\left(c^{i}\right)^{T} \underline{z}-$ $\delta \leq \delta-r$, and hence the convex body $K$ is contained in the parallelepiped $\left[x \in E^{d} \mid\left(c^{i}\right)^{T} \beta \underline{z}^{i} \leq\left(c^{i}\right)^{T} x \leq\left(c^{i}\right)^{T} \beta \bar{z}^{i}, 1 \leq i \leq d\right\}$. Again, from (2.1), we get

$$
V(K) \leq \beta^{d} \cdot\left|\operatorname{det}\left(y^{1}, \ldots, y^{d}\right)\right| \text {. }
$$

On account of the choice of $\delta$ we have, by (3.1) and (3.2), the bound

$$
\bar{V}(K) / \underline{V}(K) \leq d !(1+\varepsilon)^{d}
$$

Next we consider the running time of the algorithm. To this end let $\langle>$ denote the numbers of bits needed to write down a rational object [GLS, p. 30]. The size of the input of the algorithm is $\langle K, \varepsilon\rangle=d+\langle r\rangle+\langle R\rangle+\langle\varepsilon\rangle$. Step [3] of the algorithm can be done with the WOPT in oracle polynomial time with respect to the input size $d+\langle r\rangle+\langle R\rangle+\langle\delta\rangle+\left\langle c^{i}\right\rangle$. The size of the output of the WOPT oracle depends on the precision needed by the WOPT to carry out its arithmetic operations. As pointed out in [GLS] the number of binary digits which are needed by the WOPT is a polynomial in $d+\langle r\rangle+\langle R\rangle+\langle\delta\rangle$ and hence a polynomial in $\langle K, \varepsilon\rangle$. This means that all the calculated points $\bar{z}^{i}, \underline{z}^{i}$ are of a fixed size and by using the well-known Gaussian elimination we can find, in polynomial time with respect to $\langle K, \varepsilon\rangle$, a vector $c$ which is orthogonal to $L\left(y^{1}, \ldots, y^{i}\right)$. In particular, the size of $c$ is bounded by a polynomial in $\langle K, \varepsilon\rangle$. If we use a suitable normalization to get $\|c\| \geq 1$, we see that we can find appropriate directions $c^{i}$ in polynomial time. Since the sizes of these directions are bounded by a polynomial in $\langle K, \varepsilon\rangle$, the running time of the WOPT is also bounded by a polynomial in $\langle K, \varepsilon\rangle$. So we have an oracle-polynomial-time algorithm.

Remarks. (1) Using binary search it can easily be seen that each WOPT in step [3] of our algorithm can be solved by at most $\log _{2}\left(3\left\|c^{i}\right\| R / \delta\right)$ calls of the WVIOL. Hence, if we take the special normalization $\left\|c^{i}\right\|_{\infty}=1$ we obtain that the running time of our algorithm is dominated by at most

$$
2 d \log _{2}\left(\frac{3 \sqrt{d} R}{r}\right)+2 d \log _{2}\left(\frac{6}{\varepsilon}+1\right)
$$


calls of the WVIOL. This shows that the running time of our algorithm-measured in the number of calls of the WVIOL - is approximately $1 / d^{2}$ of the running time of the algorithm of Applegate and Kannan.

(2) If we take, for example, as direction $c^{i+1}, 1 \leq i \leq d-1$, the vector which is orthogonal to $L\left(y^{1}, \ldots, y^{i}, e^{i+2}, \ldots, e^{d}\right)$ and satisfies $c_{i+1}^{i+1}=1$, we do not need to compute $\operatorname{det}\left(y^{1}, \ldots, y^{d}\right)$, since

$$
\left|\operatorname{det}\left(y^{1}, \ldots, y^{d}\right)\right|=\prod_{i=1}^{d}\left(c^{i}\right)^{T} y^{i}
$$

\section{References}

[AK] D. Applegate, R. Kannan, Sampling and Integration of Near Log-Concave Functions, Computer Science Department Report, Carnegie-Mellon University, 1990.

[BF] I. Bárány, Z. Füredi, Computing the volume is difficult, Discrete Comput. Geom. 2 (1987), 319-326.

[BH] U. Betke, M. Henk, Estimating Sizes of a Convex Body by Successive Diameters and Widths, Mathematika 39 (1992), 247-257.

[BoF] T. Bonnesen, W. Fenchel, Theorie der konvexen Körper, Springer-Verlag, Berlin, 1934.

[DF] M. Dyer, A. Frieze. Computing the Volume of Convex Bodies: A Case Where Randomness Provably Helps, Computer Science Department Report, Carnegie-Mellon University, 1991.

[DFK] M. Dyer, A. Frieze, R. Kannan, A random polynomial-time algorithm for approximating the volume of convex bodies, J. Assoc. Comput. Mach. 38 (1991), 1-17.

[E] G. Elekes, A geometric inequality and the complexity of computing the volume. Discrete Comput. Geom. 1 (1986), 289-292.

[GLS] M. Grötschel, L. Lovász, A. Schrijver, Geometric Algorithms and Combinatorial Optimization, Springer-Verlag; Berlin, 1988.

[YN] D. B. Yudin, A. S. Nemirovskiî, Informational complexity and efficient methods for the solution of convex extremal problems, Ėkonom. i Mat. Metody 12 (1976), 357-369 (Russian) (English translation, Matekon 13(3) (1977), 25-45).

Received November 15, 1991. 